\title{
Correspondence
}

\section{A computerised lithium clinic diary system}

\section{DeAR SIRS}

The concept of a centralised register of patients maintained on lithium was adopted in order to ensure regular monitoring and reliable recording of serum lithium levels. Early identification of defaulters is made simple so that appropriate reminders may be sent or more direct follow up undertaken as required. Computerisation of such a recording and management system has obvious advantages. It has been possible to achieve a high degree of automation in respect of patient notification of due blood tests, and staff notification of defaulters.

The system essentially uses a 'loop' mechanism. Upon entry of a serum lithium result, the operator selects an interval varying from one to eight weeks when the next test is due. From this interval the computer selects the next due date and a letter is issued automatically approximately one week prior to that date, allowing the patient time to attend for blood sampling. The computer then 'waits' for the result to be entered, allowing two weeks 'grace' from the due date of blood sampling. If no result is entered by that time the user is made aware and a reminder letter issued.

The system may be represented diagrammatically as illustrated.

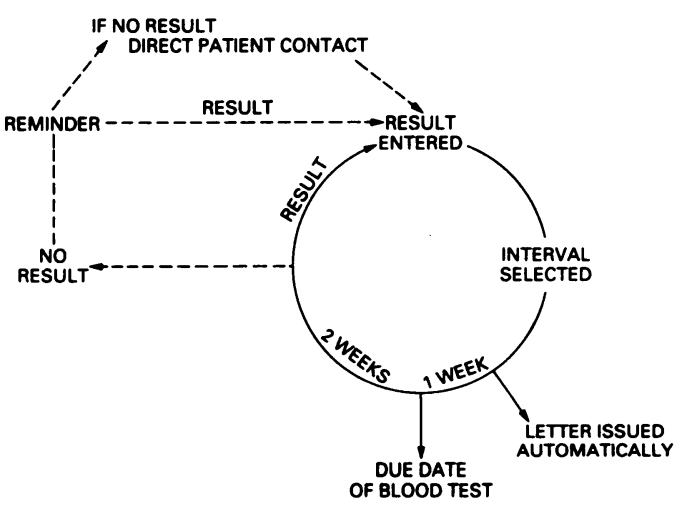

The diary system was developed through collaboration between clinician and computer programmer and now comfortably serves a district of approximately 200,000 . Indeed the facility remains somewhat underused and could easily manage a population of lithium takers from a much larger district. It has obviated the need for tedious and time consuming manual recording, patient notification and organisation of records.

The system is designed to run on any IBM compatible machine, using dBase for programming environment. Due to the confidential nature of the information stored the system is password protected and so access is strictly limited to authorised users. Simplicity of operation has been a key factor in the programme development. Thus, day to day tasks such as input of results, changes to client data, the issuing of letters and identification of defaulters, are effected from a simple 'onscreen' menu.

Routine operations are carried out on a daily basis by medical secretaries, on average taking about ten minutes. An historical record of all information is kept automatically, which in the longer term will have useful research applications.

Staff training in all aspects of the programme operation took no more than one day. The system has now been successfully in operation for approximately six months, appears to be well understood and accepted by the patients concerned and is providing highly advantageous in terms of ongoing clinical management.

Paul McMahon ANDREW BROWN

Great Yarmouth and Waveney Health Authority Great Yarmouth, Norfolk

\section{Assessment of parasuicide}

DeAR SirS

I would like to point out to trainees embarking on the assessment of parasuicide that there are alternatives to Dr Flanigan's philosophy (Bulletin, July $1988,12,284-285$ ) which they may find more rewarding.

I agree with Dr Flanigan that only a small proportion of parasuicides have clear-cut mental illness. Of the rest, however, many have depressive 
symptoms, some have a few of the 'textbook' risk factors for completed suicide but almost all are in emotional distress. If one adopts Dr Flanigan's 'screening' rationale one is indeed left with making arbitrary judgements on this group.

Surely Dr Flanigan is wrong and the 'ethical guidelines' for dealing with this group are clear. They are people who are suffering and as psychiatrist, physician and fellow human one should aim to alleviate their distress in whatever ways are possible. Before we can attempt this we need to sense why the patient felt driven to parasuicide. A warm empathic approach in a quiet room off the main ward and ensuring that the patient has fully recovered from the effects of alcohol or sedative drugs are essential to any worthwhile assessment. A system for understanding emotional disturbance and suicidal behaviour, such as that outlined by Beck $^{1}$ not only enhances the assessment but may also help the patient to feel understood. Such an approach allows for therapeutic intervention during the assessment.

The assessment of parasuicide is an opportunity for intervention in a group of patients at risk of further self-harm and completed suicide, many of whom may never be able to articulate or cope with their distress in any other way. The defensive "screening' approach may help the trainee to sleep easier and keep the demand for beds down but it will do little to alter such patient's maladaptive reactions to emotional stress.

A desire to understand the individual attempters actions is not only humane, it will help us unravel the aetiology of suicidal and parasuicidal behaviour. This is an area, as any psychiatric textbook chapter on suicide will show, where our knowledge is grossly deficient.

St Thomas' Hospital

P. M. MCLAREN London $S E 1$

\section{Reference}

${ }^{1}$ BECK, A. et al (1979) Specific techniques for the suicidal patient. In Cognitive Therapy of Depression. New York: The Guildford Press.

\section{Training in psychotherapy}

Dear Sirs

Professor Marks (Bulletin, June 1988) has made several very pertinent suggestions for improving the quality and balance of higher psychotherapy training. I would like to single out two of his suggestions for further comment. Firstly, he proposes a greater emphasis on and familiarity with the empirical basis of psychotherapy; for example a thorough knowledge of the indications for the various forms of psychotherapy and the routine application of outcome measures to the trainee's clinical practice. This suggestion is vital for the long term health of psychotherapy as a sub speciality. Only through first hand knowledge of such issues will the trainee acquire a critical appreciation of the strengths and weaknesses of his speciality and of his own clinical practice. This awareness could provide a powerful stimulus to the development of clinically relevant research ideas and a commitment to evaluation. The paucity of relevant good quality UK based psychotherapy research is painfully apparent in the journals and at conferences such as those of the Society for Psychotherapy Research and attests to the vital need for developments such as Professor Marks suggests.

How can this training requirement be best met? Probably very few training centres can provide the broad academic and clinical input required. There is consequently an obvious role for the College to fulfil, possibly by mounting specialised research methodology workshops in psychotherapy, similar in style to the successful workshops for general psychiatry training already in existence. Here experts on psychotherapy research could sharpen up the trainee's awareness of the indications for the various therapies, give indications of the key areas currently requiring research in psychotherapy, and the various levels at which trainees could contribute to this research. In addition advice could be given on how to apply measures to the trainee's clinical work and which measures best to apply. The College might like to consider at this point establishing a co-ordinated national research programme in psychotherapy as this would have numerous benefits, including reducing the likelihood of a great deal of redundancy and irrelevancy in terms of the research individual trainees might undertake.

The second recommendation Professor Marks makes is that trainees should be able to apply and supervise most of the methods of psychotherapy. There are good clinical grounds for supporting this recommendation and ensuring that the psychotherapist is able to respond flexibly within and between patients in terms of the therapeutic approaches he is able to apply. Individual trainers, however, are unlikely to be able to satisfy these eclectic needs and the College will therefore probably have to consider establishing rotational schemes which might need to be sub-regional rather than district-based if they were to meet the expanded needs for training.

Furthermore, an adequate training in family therapy, marital therapy, behaviour therapy and the treatment of sexual dysfunction, if it were to be contained within the current four year time limitation, would necessarily impinge on the time currently 